\title{
Arquitectura de la información en los entornos virtuales de aprendizaje. Aplicación de la técnica card sorting y
análisis cuantitativo de los resultados
} Artículo

\author{
Por Yusef Hassan Montero, Francisco Jesús Martín Fernández, \\ Dunia Hassan Montero y Óscar Martín Rodríguez
}

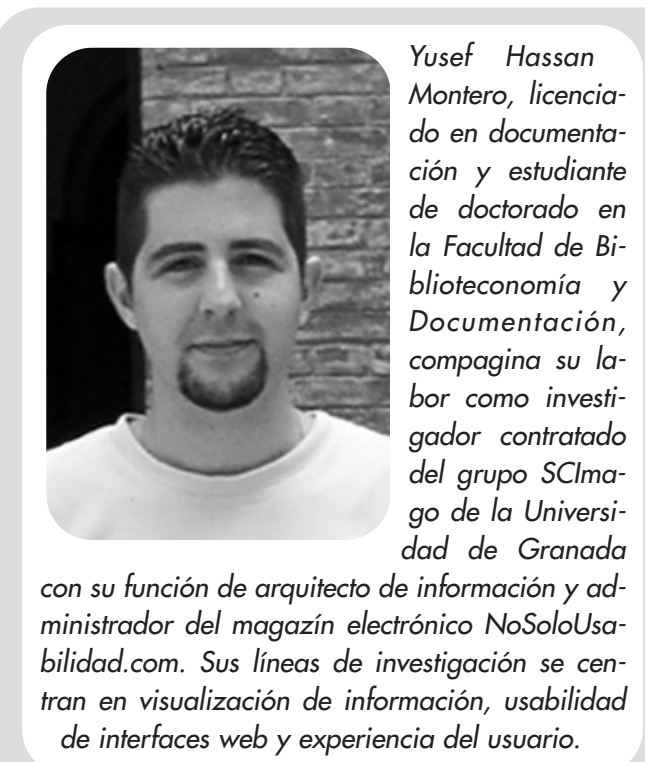

Resumen: La organización de la información de un espacio web será más útil cuanto más adaptada se encuentre al modelo mental de sus usuarios. Este requerimiento es aún de mayor importancia en entornos virtuales de aprendizaje, donde el grado de facilidad de uso favorece o dificulta la asimilación de contenidos por parte del alumno. Para esto, es imprescindible adoptar técnicas y métodos de diseño centrado en el usuario en el

\section{Francisco Jesús} Martín Fernández, diplomado en biblioteconomía y licenciado en documentación, trabajó en Baratz Servicios de Teledocumentación hasta incorporarse como personal técnico del $\mathrm{Ce}$ vug (Centro de Enseñanzas Virtuales de la Universidad de Granada). Sus líneas de

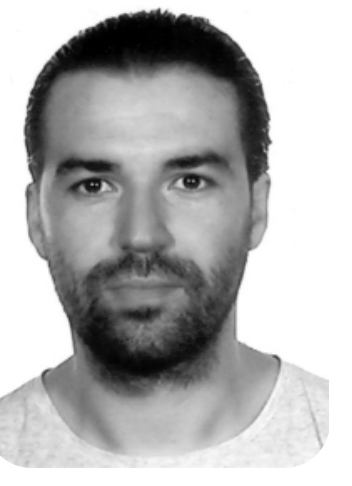
investigación se centran en usabilidad, accesibilidad y arquitectura de la información en entornos e-learning. Además es administrador y diseñador del magazín electrónico NoSoloUsabilidad.com.

proceso de creación de entornos web usables, como es el caso de la técnica de card sorting. En este trabajo presentamos la aplicación de card sorting de tipo "abierto" en el marco de un proyecto de e-learnig del Centro de Enseñanzas Virtuales de la Universidad de Granada (Cevug). Para realizar el estudio se contó con sesenta participantes, a cada uno de los cuales se le proporcionó un conjunto de doce tarjetas etiquetadas con las categorías que incorpora la plataforma de teleformación WebCT. Los resultados han sido analizados cuantitativamente mediante diferentes técnicas estadísticas: clustering o técnicas de cluster y MDS (escalamiento multidimensional).

Palabras clave: Arquitectura de la información, Card sorting, Técnicas de clustering, MDS, E-learning, Usabilidad, Diseño centrado en el usuario.

Dunia Hassan Montero, licenciada en ciencias políticas y sociología, cursó el doctorado en el Instituto de la Paz y los Conflictos y fue becaria de investigación del Centro de Enseñanzas Virtuales de la Universidad de Granada (Cevug). Trabajó en Extremadura como técnica del proyecto europeo Infodex (Estrategia Re-

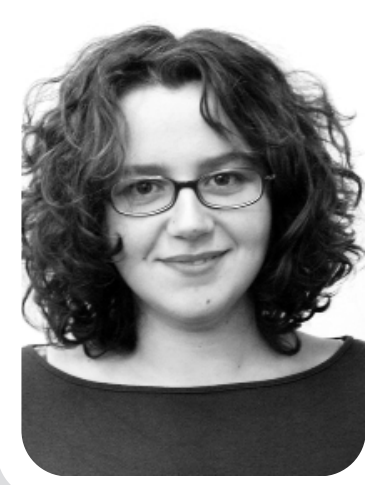
gional de Sociedad de la Información). Actualmente realiza un máster de periodismo audiovisual (Universidad Rey Juan Carlos, Madrid) y se especializa en documentación para medios de comunicación (lepala-Comunidad de Madrid).
Title: Information architecture in e-learning environments: application of the card-sorting technique and quantitative analysis of the results

Abstract: The organisation of information in a web site is more useful if it adapts to the way the user thinks and behaves. This is especially important in e-learning sites, where usability is directly linked to the way the user learns. The best way to
Óscar Martín Rodríguez, diplomado en biblioteconomía y licenciado en documentación por la Universidad de Granada, trabajó durante dos años en Baratz Servicios de Teledocumentación, en el Departamento de Asistencia Técnica de Absys. En la actuali-

dad forma parte del personal técnico del Cevug (Centro de Enseñanzas Virtuales de la Universidad de Granada) y cursa estudios de doctorado, siendo sus líneas de investigación los estándares en el e-learning.

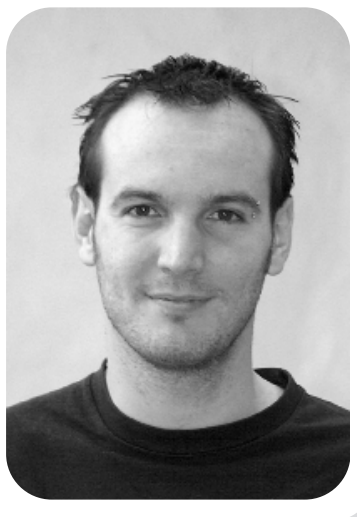


manage an intuitive and easy-to-use web site it is to organise the site as the student would: that is, following the way the user thinks and manages information. One method is to apply the card sorting technique based on observation of how the users form groupings and associate a predetermined number of categories. In this paper the authors present the results of applying this technique to an e-learning project at the University of Granada (Cevug). Sixty people were studied as they organised twelve categories included in the WebCT platform and a quantitative analysis was made of the results by different statistical techniques: clustering and MDS.

Keywords: Card sorting, Multidimensional scaling, Clustering analysis, E-learning, Information architecture, Usability.

Hassan Montero, Yusef; Martín Fernández, Francisco Jesús; Hassan Montero, Dunia; Martín Rodríguez, Óscar. "Arquitectura de la información en los entornos virtuales de aprendizaje. Aplicación de la técnica card sorting y análisis cuantitativo de los resultados”. En: El profesional de la información, 2004, marzo-abril, v. 13, n. 2, pp. 93-99.

\section{Introducción}

La arquitectura de la información es un campo de estudio que aparece a partir de la necesidad de dar solución a problemas derivados de la organización y estructuración de grandes volúmenes de información.

El surgimiento de la web y la popularización del hipertexto, la complejidad de los nuevos sistemas de información, así como la gran diversidad de usuarios y contextos de uso, han originado la necesidad de hacer frente a nuevos retos de diseño a los que ninguna disciplina actual puede dar solución por sí sola (Rosenfeld, 2002). Es por ello por lo que ha sido en la era web cuando la arquitectura de la información ha experimentado su mayor auge. Su aplicación en un sitio web (entendida como el resultado de la actividad de clasificar, describir, estructurar y etiquetar sus contenidos) si bien no es percibida directamente por el usuario, como indica Gullikson (1999), tiene un claro impacto en su usabilidad, es decir, en la eficacia, eficiencia y satisfacción de uso. Usabilidad y arquitectura de la información no son lo mismo (Lash, 2002), aunque para los fines de este trabajo serán englobadas en una visión "amplia" de la segunda, la cual, por su carácter multidisciplinar, se nutre de técnicas, metodologías y teorías de una gran variedad de áreas de conocimiento (diseño gráfico, psicología cognitiva, ciencias de la documentación, etc.) así como de prácticas profesionales y estudios de casos reales.

Entre las aportaciones metodológicas que ha recibido por parte de disciplinas como la interacción persona-ordenador (HCI, human-computer interaction) cabe destacar el diseño centrado en el usuario (DCU) (Norman; Draper, 1986). Se trata de un marco metodológico en el que se asume que las características, necesidades y objetivos del usuario deben ser las que conduzcan todo el proceso de diseño. El DCU se basa en un continuo e iterativo proceso de "diseño-prototipado-evaluación" lo que permite, desde tempranas etapas del desarrollo, evaluar lo realizado y por tanto validar la arquitectura del producto.
El DCU, además de proporcionar métodos para la evaluación y el prototipado de lo diseñado, también ofrece un conjunto de técnicas para la toma de decisiones por parte del arquitecto de información sobre qué diseño y organización de la información resultaría más acorde con las necesidades y características del usuario. Entre estas técnicas se encuentra la de card sorting, que se basa en la observación de cómo los usuarios agrupan y asocian entre sí un número predeterminado de tarjetas etiquetadas con las diferentes categorías temáticas del sitio web. De esta forma, partiendo del comportamiento de los propios usuarios, es posible organizar y clasificar la información de un espacio web conforme a su modelo mental. Esta técnica, además de en DCU, ha sido utilizada con éxito durante años con otros fines, tales como en psicología clínica (Obonsawin, et al., 1999) o en la adquisición de conocimiento en sistemas expertos (Wagner; Najdawi; Chung, 2001).

\section{«El card sorting se basa en la observación de cómo los usua- rios agrupan y asocian entre sí un número predeterminado de tarjetas etiquetadas con las di- ferentes categorías temáticas del sitio web»}

En este trabajo presentamos la aplicación de card sorting de tipo "abierto" en el marco de un proyecto de e-learnig del Centro de Enseñanzas Virtuales de la Universidad de Granada (Cevug), realizando un análisis cuantitativo de los resultados a través de diferentes técnicas estadísticas: clustering o técnicas de cluster (Martín, 1999) y MDS (escalamiento multidimensional). La estructura seguida en el artículo es la siguiente: (2) contextualización del estudio en el marco de los entornos virtuales de aprendizaje; (3) explicación de los tipos de card sorting y análisis; (4) metodología seguida en el estudio; (5) interpretación de los resultados obtenidos; (6) discusión acerca de las ven- 
tajas de aplicar la metodología propuesta en este trabajo; y (7) conclusiones y trabajos futuros.

\section{Formación virtual}

Uno de los obstáculos que subyacen en los nuevos modelos de aprendizaje a través de entornos virtuales tiene que ver con la distancia que se establece entre el usuario y el medio web a través del cual interactúa y aprende. Este entorno debe ser intuitivo y fácil de usar. El estudiante en contextos ricos en información, como es la web, debe encontrar de forma rápida y lógica las herramientas y recursos que se le ofrecen, más útiles y eficaces cuanto más se asemejen al modo en que él habitualmente razona y actúa.

Al mismo tiempo, el entorno virtual posibilita que todos los participantes en un curso en línea (autores, profesores, tutores y estudiantes) puedan disfrutar de las características propias del nuevo marco tecnológico, tales como el aumento de las posibilidades de comunicación entre los participantes (independientemente del momento y lugar desde el que accedan al curso); la fluidez del intercambio de información y la diversificación de fuentes y formatos de los contenidos.

Teniendo en cuenta los actores y elementos que intervienen en la enseñanza virtual, podemos establecer entre ellos el siguiente tipo de relaciones:

-Alumno-contenidos teóricos: como en la enseñanza tradicional, se trata de que el alumno lea, reflexione y asimile la información e ideas proporcionadas a través del medio electrónico.

-Alumno-tutor: es imprescindible en la enseñanza virtual, mientras que en la tradicional se le tiende a restar importancia. Nos referimos a la relación entre el tutor y los alumnos sobre la información e ideas presentadas.

-Alumno-alumno: la interacción entre ellos es primordial en la metodología de aprendizaje participativo que se intenta fomentar a través de los nuevos entornos de enseñanza virtual. Las herramientas de comunicación posibles a través de internet son una nota distintiva de la mera enseñanza a distancia asistida.

-Alumnos-actividades: la establece el alumno con los contenidos teóricos, el tutor y el resto de alumnos a través de las diversas tareas programada en un curso virtual.

Como se puede observar, todas implican un medio y unos contenidos adaptados y específicos, es decir, que favorecen el correcto flujo de interacciones. En lo que al contenido se refiere, los especialistas hablan de "diseño instructivo" para referirse al conjunto de factores que contribuyen a que se mantenga cierta coherencia didáctica y organizativa (Moreno; Bailly-Bai- llière, 2000) es decir, a la transformación de lo que podría ser un simple texto electrónico en un recurso hipertextual adecuadamente organizado y temporalizado, donde exista un equilibrio entre los recursos de aprendizaje, ayudas al estudio, actividades y sistemas de autoevaluación.

En cuanto al medio, la propia experiencia de la tele-enseñanza ha evidenciado que éste, su arquitectura e interfaz, garantiza, o por el contrario impide, la asunción de los contenidos por parte del alumno. Así, de la inicial preocupación por diseñar contenidos que se adaptasen al medio digital, hemos pasado a interesarnos por cómo éste se adapta a las necesidades del alumno.

\section{«De la inicial preocupación por diseñar contenidos que se adaptasen al medio digital, he- mos pasado a interesarnos por cómo éste se adapta a las ne- cesidades del alumno»}

Por lo tanto podemos concluir que mejorando la interacción entre alumno y medio no sólo se favorecerá la asimilación de contenidos por su parte sino también la comunicación o interacción entre actores, garantizando una experiencia de aprendizaje satisfactoria.

\section{Card sorting: tipología y análisis}

Esta técnica nos permite, desde las primeras etapas del proceso de diseño, anticipar cuál será la organización de categorías o menús de navegación que mejor se adapte al modelo mental de los usuarios. Es una técnica de elicitación u obtención de información de los sujetos participantes en el estudio. Atendiendo a la clasificación de Rosenfeld y Morville (2002) podemos diferenciar entre dos tipos: abierto y cerrado. En el primero el usuario puede agrupar las categorías libremente en el número de conjuntos que crea necesario mientras que, en el cerrado, los grupos o conjuntos están predefinidos y etiquetados, con lo que el usuario únicamente deberá colocar cada categoría en donde crea que corresponda. Este segundo tipo está recomendado para verificar si un diseño de información es familiar y comprensible para el usuario, mientras que el abierto tiene el objetivo de descubrir qué tipo de clasificación de categorías sería más correcto utilizar.

La cantidad y calidad de la información que podamos extraer del empleo de esta técnica dependerán del tipo de análisis que realicemos. Entre los tipos de análisis, es posible distinguir entre: 
-Cualitativo: el responsable de realizar la prueba prestará especial atención a todos los aspectos relativos a cómo cada usuario en concreto organiza las tarjetas, qué problemas tiene para realizar la tarea, qué categorías agrupa inmediata e intuitivamente y sobre cuáles duda más, qué preguntas hace durante la prueba, etc.

-Cuantitativo: se basa en la aplicación de técnicas estadístico-automáticas sobre los grupos resultantes de la agrupación realizada por los usuarios.

Aunque el análisis cualitativo puede ofrecer información adicional al cuantitativo, el número de participantes en la prueba debe ser necesariamente bastante reducido con el fin de poder realizar un seguimiento de cada uno de ellos y su modo de actuar. Esto implica una pérdida en la representatividad de los resultados y por lo tanto en su validez. En el cuantitativo, puesto que el número de participantes será bastante más elevado, se hace imprescindible el uso de técnicas estadísticas que faciliten la comprensión e interpretación de los resultados, normalmente a través de representaciones gráficas que permitan visualizar las relaciones de agrupación y distancia entre categorías.

\section{Metodología}

\subsection{Proceso o procedimiento.}

Para realizar el estudio se ha contado con 60 participantes, número significativamente superior al de otros estudios (Upchurch; Rugg; Kitchenham, 2001), (Nielsen; Sano, 1994) o (Cooper, 2002) lo que favorece la validez del presente trabajo.

A cada uno de los participantes se le proporcionó un conjunto de 12 tarjetas etiquetadas con las siguientes categorías: Guía de estudio; Descarga de materiales; Foro; Web personal; Temario; Glosario; Correo; Autoevaluación; Búsquedas; Mi progreso; Chat y Actividades.

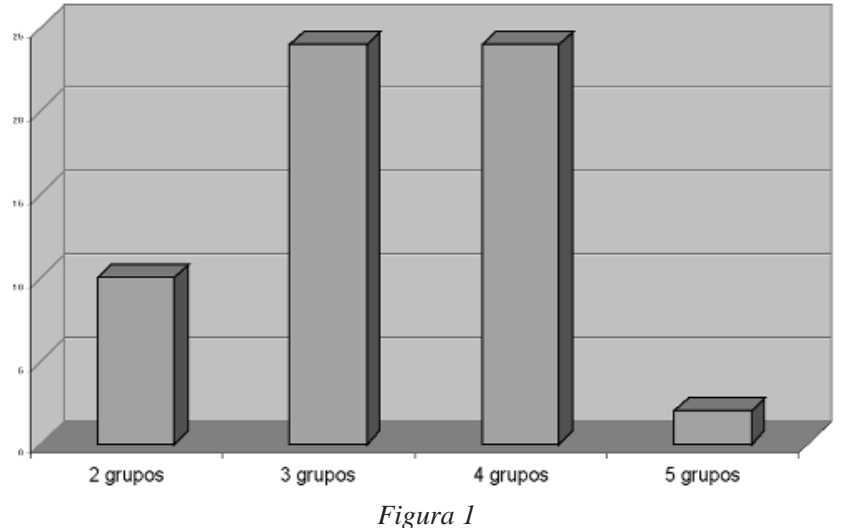

Las categorías responden a las que ofrece la plataforma de tele-formación WebCT ${ }^{1}$, plataforma virtual que ha escogido la Universidad de Granada al considerar que incorporaba todas las funcionalidades necesarias para poder desarrollar la labor docente y administrativa de los cursos virtuales, tales como la generación de materiales multimedia; la provisión de materiales de apoyo a través de índices o glosarios; la comunicación vía correo electrónico, foros de discusión, chat y pizarra electrónica y, finalmente, sistemas de evaluación y autoevaluación. Los participantes agruparon las diferentes tarjetas en tantos grupos como creyeron necesarios. Al comienzo de la prueba se les explicó que el criterio de agrupación debía ser "por similaridad".

Se fijó un tiempo máximo para realizarla de 10 minutos, sin contabilizar el destinado por los responsables a su explicación, en la que se hizo especial hincapié sobre cuáles eran los objetivos de la misma y que éstos nunca eran la evaluación de los propios participantes. Los colaboradores y sus perfiles (experiencia en internet, nivel académico, edad, etc.) fueron seleccionados de acuerdo con características que se corresponden con el tipo de usuario potencial del sistema.

\begin{tabular}{|c|c|c|c|c|c|c|c|c|c|c|c|c|}
\hline & $\begin{array}{l}\begin{array}{l}\text { Guía de } \\
\text { estudio }\end{array} \\
\end{array}$ & $\begin{array}{c}\text { Descarga de } \\
\text { materiales }\end{array}$ & Foro & $\begin{array}{c}\text { Web } \\
\text { personal }\end{array}$ & Temario & Glosario & Correo & $\begin{array}{c}\text { Auto- } \\
\text { evaluación }\end{array}$ & Búsquedas & Mi progreso & Chat & Actividades \\
\hline Guía de estudio & 58 & 30 & 1 & 1 & 47 & 49 & 1 & 24 & 17 & 22 & 1 & 21 \\
\hline $\begin{array}{l}\text { Descarga de } \\
\text { materiales }\end{array}$ & 30 & 57 & 6 & 9 & 40 & 36 & 6 & 16 & 19 & 13 & 6 & 13 \\
\hline Foro & 1 & 6 & 59 & 33 & 0 & 1 & 40 & 1 & 21 & 1 & 54 & 9 \\
\hline Web personal & 1 & 9 & 33 & 56 & 0 & 0 & 42 & 5 & 22 & 6 & 32 & 3 \\
\hline Temario & 47 & 40 & 0 & 0 & 60 & 55 & 0 & 21 & 17 & 15 & 0 & 18 \\
\hline Glosario & 49 & 36 & 1 & 0 & 55 & 60 & 1 & 20 & 17 & 17 & 0 & 20 \\
\hline Correo & 1 & 6 & 40 & 42 & 0 & 1 & 57 & 0 & 19 & 1 & 43 & 5 \\
\hline Autoevaluación & 24 & 16 & 1 & 5 & 21 & 20 & 0 & 60 & 8 & 51 & 0 & 30 \\
\hline Búsquedas & 17 & 19 & 21 & 22 & 17 & 17 & 19 & 8 & 60 & 7 & 20 & 17 \\
\hline Mi progreso & 22 & 13 & 1 & 6 & 15 & 17 & 1 & 51 & 7 & 57 & 0 & 33 \\
\hline Chat & 1 & 6 & 54 & 32 & 0 & 0 & 43 & 0 & 20 & 0 & 58 & 7 \\
\hline Actividades & 21 & 13 & 9 & 3 & 18 & 20 & 5 & 30 & 17 & 33 & 7 & 59 \\
\hline
\end{tabular}

Tabla 1 
La prueba fue realizada por los usuarios de forma manual. Aunque existen aplicaciones software - tanto independientes ${ }^{2}$ como basadas en web (Wood; Wood; Anderson, 2002) - para realizar card sorting virtualmente y que nos hubieran facilitado la posterior cuantificación, procesamiento y análisis automático de los resultados, creemos que suponen en sí una barrera para los usuarios, quienes no manejan las tarjetas con la misma facilidad y comodidad que de forma manual debido a la propia problemática inherente a la interacción persona-ordenador.

\subsection{Resultados.}

Los usuarios agruparon las 12 categorías en un número de entre 2 y 5 grupos diferentes, siendo la media de grupos creados por participante de 3,3, y tanto la moda como la mediana 3 . En la figura 1 podemos observar la frecuencia del número de grupos de categorías realizadas por los usuarios.

El porcentaje medio de veces que un usuario, en el tiempo proporcionado para la tarea, no ha sabido dónde colocar una categoría, es del 2,6\%, cifra claramente despreciable. Si hubiera sido significativamente superior se debería cuestionar la idoneidad del rotulado de las categorías ya que probablemente se estaría usando un lenguaje poco familiar para los usuarios.

Estos resultados han sido representados electrónicamente, especificando por cada usuario el número de grupos realizados y la correspondencia de cada categoría a cada uno de estos grupos.

\subsection{Análisis cuantitativo: clustering y $M D S$.}

Mediante un script en PHP contabilizamos, sobre la representación electrónica de los datos, las co-ocurrencias entre categorías, esto es, el número de veces que cada par de categorías han sido colocadas en un mismo grupo por los usuarios, obteniendo como resultado la tabla 1. Para realizar el tratamiento estadístico de los datos se ha optado por utilizar el paquete estadístico $\mathrm{Spss}^{3}$. Cabe aclarar que se podría haber hecho uso de cualquier otro de última generación, ya que la mayoría ofrece en la actualidad las herramientas y algoritmos necesarios para la aplicación de las técnicas de análisis presentadas en este trabajo. Los datos son introducidos tal como aparecen en la tabla 1, ya que Spss posibilita el cálculo, necesariamente previo a la aplicación de los algoritmos, de las (di) similaridades entre casos o variables, en este trabajo representados por las categorías.

El primer análisis que realizamos sobre los datos es el denominado análisis de clustering o conglomerados, eligiendo la técnica de Ward entre todas las posibles. Este método, conocido también como el de la suma de cuadrados se caracteriza, y por tanto diferencia

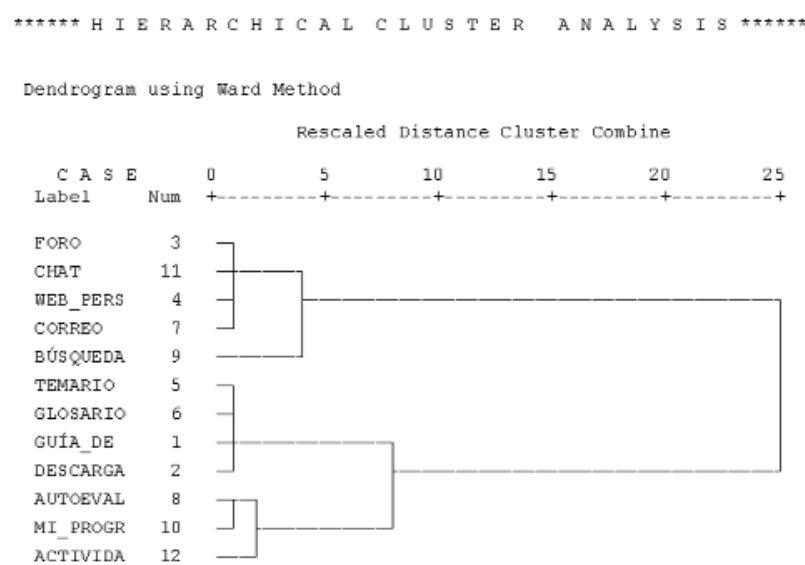

Figura 2

del resto, por minimizar el tamaño de las ramas (categorías) maximizando el árbol (estructura o grupos de categorías) de la jerarquía de conglomerados resultante (Herrero Solana; Moya Anegón, 2001). Es por este comportamiento por el que fue elegido, ya que las relaciones entre categorías serán observadas a través de otra técnica de análisis (MDS), mientras que con el clustering lo que pretendemos es observar las relaciones entre grupos de categorías. Tras la aplicación del algoritmo de clustering, obtenemos la representación gráfica en forma de dendograma de la figura 2.

En este tipo de representación se pueden apreciar claramente las relaciones de agrupación entre categorías e incluso entre grupos de ellas, pero no así las relaciones de cercanía o similaridad entre categorías, principalmente entre aquellas que pertenecen a grupos diferentes.

Es por ello por lo que además aplicamos sobre los datos el algoritmo de $M D S$, técnica de análisis multivariante cuyo objetivo es doble:

-Extraer la estructura subyacente de los datos a analizar (objetivo compartido por las técnicas de clustering).

- Obtener una representación geométrica de los mismos en un espacio de dimensionalidad reducida (2D ó 3D) de forma que el resultado sea interpretable por simple inspección visual.

Tras aplicar el algoritmo obtenemos la representación gráfica bidimensional de la figura 3, en la que podemos observar las mencionadas relaciones de cercanía o similaridad entre categorías, de difícil apreciación a través de la figura 2 .

Como indican Guerrero y Ramírez (2002), la técnica de $M D S$ puede ser utilizada como alternativa a otras multivariantes o, como es nuestro caso, como complemento a las mismas ya que tanto la técnica de clustering como $M D S$ ofrecen sus propias ventajas para la interpretación visual de los resultados. 


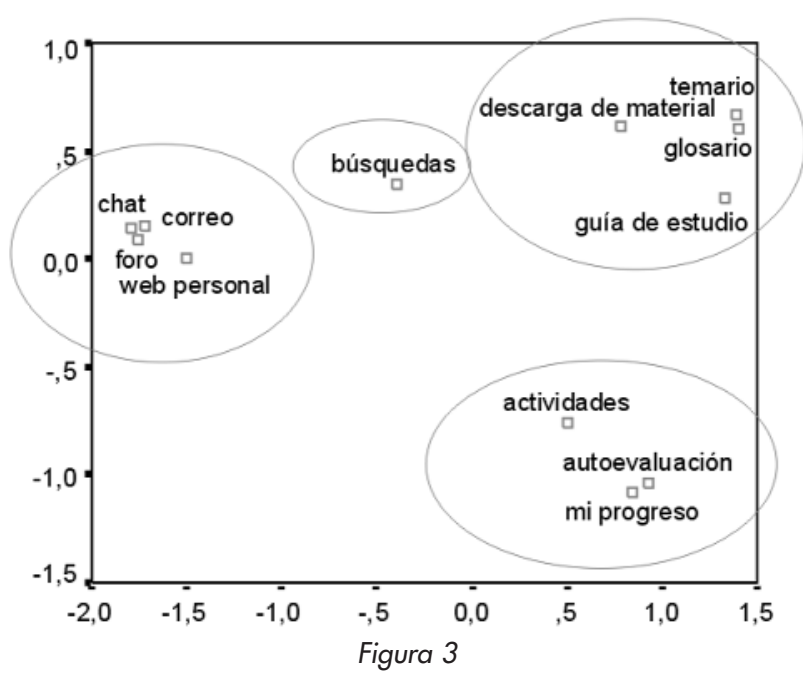

\section{Interpretación de los resultados}

Como se puede observar en las representaciones gráficas, finalmente hemos obtenido 3 grupos claramente diferenciados:

- Glosario, Temario, Descarga de materiales y Guía de estudio.

-Chat, Correo, Web personal y Foro.

—Actividades, Mi progreso y Autoevaluación.

La categoría Búsquedas, aunque más cercana a los dos primeros grupos (principalmente al primero) que al tercero, constituye por sí sola uno independiente ya que no se ve incluida en ninguno de los anteriormente mencionados. Así, cada uno de ellos podemos etiquetarlos manualmente de acuerdo a sus categorías contenidas, con los siguientes rótulos: Materiales de estudio, Comunicación, Personal y Búsquedas.

Aunque el objetivo del estudio de la ordenación de categorías por los participantes es reducir las dificultades de navegación y localización de las herramientas, no deja de ser curioso el hecho de que hayan agrupado la etiqueta Actividades junto a las de Autoevaluación y Mi progreso. Sin duda, el usuario educado en entornos de enseñanza tradicional continúa considerando las actividades como un instrumento evaluador y no como un recurso activo para ofrecer y asumir contenidos, idea ésta que sustenta la enseñanza virtual.

El hecho, además, de que la Descarga de materiales permanezca junto a Glosario y Temario, todas ellas agrupadas bajo la rúbrica Materiales de estudio, nos hace reflexionar acerca de la posibilidad de que el usuario considere que lo prioritario es ver los contenidos y descargarlos (para posteriormente imprimírselos). Así, el aula virtual continuaría empleándose como medio a través del cual obtener el material necesario y no como un espacio de interacción educativa. Por otra parte es lógico teniendo en cuenta que aún son elevados los costes de conexión y la calidad de la misma también deja mucho que desear para la mayoría de los hogares españoles.

Especial atención merece también que la categoría Búsquedas no haya quedado claramente incluida en ninguno de los grupos, conformando por sí sola un grupo a medio camino entre Materiales de estudio y Comunicación. Esto es debido a que representa para el usuario una aplicación o herramienta, al igual que todas las categorías del grupo Comunicación, pero con una función claramente relacionada con los Materiales de estudio: la recuperación de información.

\section{Discusión}

El modo en que los usuarios han organizado las etiquetas nos debe servir para reflexionar acerca de la necesidad de comprobar o testar con los propios destinatarios cualquier diseño de un espacio virtual de enseñanza, más allá de las ideas preconcebidas acerca de cuál sería la óptima organización de los recursos en el aula virtual. Pero, incluso más relevante que la experiencia descrita en este trabajo es la metodología propuesta para el análisis de los resultados del card sorting, destinada a simplificar y facilitar la comprensión de éstos por el diseñador. Las técnicas de cluster y $M D S$, como se demuestra tanto en este trabajo como en estudios similares (Cooper, 2002) resultan de gran utilidad para la interpretación de resultados y se complementan perfectamente entre sí para esta tarea.

Por tanto, discrepamos de Rosenfeld y Morville (2002) cuando al hablar de las representaciones gráficas generadas mediante técnicas estadístico-automáticas a partir de resultados de card sorting opinan que son "con frecuencia bastante complejas y difíciles decomprender", ya que esta opinión se encuentra motivada por la errónea elección del tipo de análisis estadístico a realizar y su consiguiente representación gráfica. En concreto, en su trabajo parecen realizar un análisis de redes sociales sobre los participantes, el cual nos ofrece información sobre patrones de actuación y relación entre participantes, pero no sobre las relaciones de agrupación y similaridad entre categorías, que es precisamente la información que se pretende extraer mediante card sorting.

\section{Conclusiones y trabajos futuros}

El empleo de la técnica de card sorting se ha demostrado como uno de los estudios de usuarios más útiles en el diseño de entornos ricos en información. Su doble función, validar y asistir en la creación de clases o grupos de categorías, proporciona también una doble herramienta para el diseño centrado en el usuario: para la toma de decisiones en etapas de diseño conceptual (presentada en este trabajo) y para la va- 
lidación de clasificaciones en etapas de evaluación de la usabilidad.

\section{«La experiencia aquí descrita no tendría sentido si no se en- contrara contextualizada en el marco del diseño centrado en el usuario»}

Hay que señalar que la experiencia aquí descrita no tendría sentido si no se encontrara contextualizada en el marco del diseño centrado en el usuario, ya que el diseño de productos web usables depende de muchas otras prácticas y técnicas: aplicación de principios de usabilidad en el diseño; evaluación de la usabilidad, principalmente mediante métodos de inspección y de test; estudio de las necesidades y requerimientos de usuarios a través de encuestas, entrevistas y estudios etnográficos; técnicas de prototipado del producto $\mathrm{y}$, finalmente, técnicas de monitorización y seguimiento del usuario y su navegación.

En futuros trabajos se propondrán otras metodologías y técnicas, complementarias a las descritas aquí para asistir y ayudar a arquitectos de la información en el diseño de entornos complejos centrados en el usuario.

\section{Notas}

1. http://www.webct.com

2. http://www-3.ibm.com/ibm/easy/eou_ext.nsf/Publish/1876

3. http://www.spss.com

\section{Bibliografía}

Cooper, Linda $\mathbf{Z}$. "Methodology for a project examining cognitive categories for library information in young children". En: Jasist, Journal of American Society for Information Science and Technology, 2002, v. 53, n. 14 , pp. 1.223-1.231.

Guerrero Casas, F. M.; Ramírez Hurtado, J. M. "El análisis de escalamiento multidimensional: una alternativa y un complemento a otras técnicas multivariantes". En: X Jornadas Asepuma (Asociación Española de Profesores Universitarios de Matemáticas para la Economía y la Empresa), 2002.

http://www.uv.es/asepuma/jornadas/madrid/K11C.pdf

Gullikson, Shelley, et al. "The impact of information architecture on academic web site usability. En: The electronic library, 1999, October, v. 17, n. 5, pp. 293-304.
Herrero Solana, Víctor; Moya Anegón, Félix. "Bibliographic displays of web-based opacs: multivariate analysis applied to latin-american catalogues". En: Libri: international journal of libraries and information services, 2001, n. 51, pp. 67-78.

Lash, Jeff. "Information architecture is not usability". En: Digital web magazine, noviembre , 2002.

http://www.digital-web.com/columns/ianythinggoes/ianythinggoes_200211.shtml

Martin, S. Cluster analysis for web site organization Internetworking, 1999, diciembre.

http://www.internettg.org/newsletter/dec99/cluster_analysis.html

Moreno, F.; Bailly-Baillière, M. Diseño instructivo de la formación online. Barcelona: Ariel Educación, 2002.

Nielsen, J.; Sano, D. Design of Sun Web: Sun Microsystems' Intranet, 1994.

http://www.useit.com/papers/sunweb/

Norman, D. A.; Draper, S. W. (eds.). User centered system design: new perspectives on human-computer interaction. Hillsdale, NJ: Lawrence Erlbaum Associates, 1986.

Obonsawin, M. C., et al. "Performance of the modified card sorting test by normal, healthy individuals: relationship to general intellectual ability and demographic variables" En: British journal of clinical psychology, 1999, n. 38, pp. 27-41.

Rosenfeld, L. "Information architecture: looking ahead". En: Jasist, Journal of American Society for Information Science and Technology, 2002, August, v. 53, n. 10, pp. 874-876.

Rosenfeld, L.; Morville, P. Information architecture for the world wide web. $2^{\text {nd }}$ edition. Sebastapol, CA: O'Reilly, 2002. Isbn 0-596-00035-9.

Rovira, Cristòfol. "Estructuras de navegación para e-learning". En: $E l$ profesional de la información, 2002, v. 11, n. 6, nov.-dic., pp. 457-466.

Upchurch, L.; Rugg, G.; Kitchenham, B. "Using card sorts to elicit web page quality attributes". En: Ieee software, 2001, July-August, pp. 84-89.

Wagner, P. W.; Najdawi, M. K.; Chung, Q. B. "Selection of knowledge acquisition techniques based upon the problem domain characteristics of productions and operations management expert systems". En: Expert systems, 2001, May, v. 18, n. 2, pp. 76-87.

Wood, L. E.; Wood, J. R.; Anderson, J. "Web-based card sorting for information architecture". En: UPA 2002 Meeting, 2002.

http://www.acm.org/chapters/nuchi/2002/09mtg_websort/WebSort.html

Yusef Hassan Montero, Francisco Jesús Martín Fernández, Dunia Hassan Montero, Óscar Martín Rodríguez.

yusef@nosolousabilidad.com

chesco@ugr.es

dunia@ugr.es

omartin@ugr.es

\section{Próximos temas especiales}

$\begin{aligned} \text { Mayo 2004 } & \text { Usabilidad y arquitectura en la web } \\ \text { Julio 2004 } & \text { Servicios de información digital en Latinoamérica } \\ \text { tiembre 2004 } & \text { Fuentes de información sobre economía y empresa }\end{aligned}$

Los interesados pueden remitir notas, artículos, propuestas, publicidad, comentarios, etc., sobre estos temas a:

epi@sarenet.es 